\title{
Close-packed Dimers on the Line: Diffraction versus Dynamical Spectrum
}

\author{
Michael Baake • Aernout van Enter
}

Received: 7 November 2010 / Accepted: 16 February 2011 / Published online: 11 March 2011

(C) The Author(s) 2011. This article is published with open access at Springerlink.com

\begin{abstract}
The translation action of $\mathbb{R}^{d}$ on a translation bounded measure $\omega$ leads to an interesting class of dynamical systems, with a rather rich spectral theory. In general, the diffraction spectrum of $\omega$, which is the carrier of the diffraction measure, lives on a subset of the dynamical spectrum. It is known that, under some mild assumptions, a pure point diffraction spectrum implies a pure point dynamical spectrum (the opposite implication always being true). For other systems, the diffraction spectrum can be a proper subset of the dynamical spectrum, as was pointed out for the Thue-Morse sequence (with singular continuous diffraction) by van Enter and Miękisz (J. Stat. Phys. 66:1147-1153, 1992). Here, we construct a random system of close-packed dimers on the line that have some underlying long-range periodic order as well, and display the same type of phenomenon for a system with absolutely continuous spectrum. An interpretation in terms of 'atomic' versus 'molecular' spectrum suggests a way to come to a more general correspondence between these two types of spectra.
\end{abstract}

Keywords Dynamical systems $\cdot$ Diffraction spectra $\cdot$ Dynamical spectra $\cdot$ Random systems

\section{Introduction}

It is well-known $[8,25,26,30]$ that pure point diffraction and pure point dynamical spectrum, under some mild assumptions, are equivalent properties of dynamical systems of translation bounded measures on $d$-space. This type of equivalence does not extend to systems with continuous spectrum, as the example of the Thue-Morse sequences shows [17]. The

M. Baake ( $\varangle)$

Fakultät für Mathematik, Universität Bielefeld, Postfach 100131, 33501 Bielefeld, Germany e-mail: mbaake@math.uni-bielefeld.de

A. van Enter

Johann Bernoulli Institute for Mathematics and Computer Science, University of Groningen,

PO Box 407, 9700 AK Groningen, The Netherlands

e-mail: a.c.d.van.enter@ rug.nl 
corresponding dynamical system can be defined via the primitive 2-letter substitution rule $a \mapsto a b, b \mapsto b a$. It supports a unique shift-invariant probability measure. The diffraction spectrum (for the associated Dirac combs with balanced weights \pm 1 ) is purely singular continuous, while the dynamical spectrum has a non-trivial pure point part in form of the dyadic rationals. This spectral information is not reflected in the diffraction spectrum, no matter whether one works with balanced or general weights.

However, this 'missing' part can be extracted from the diffraction of a factor of the ThueMorse system, the so-called period doubling sequences, which are Toeplitz sequences (the corresponding system can be defined by the substitution rule $a \mapsto a b, b \mapsto a a$ ). For a discussion of the dynamical spectra of both systems, we refer to [27], while the discrepancy with the diffraction spectrum was noticed in [17]. The diffraction spectrum of the ThueMorse and period doubling systems (in modern terminology) is discussed in detail in [5, 7]; see also the references given there for previous work.

Below, we discuss a simple system that displays a similar phenomenon in the presence of absolutely continuous diffraction. We employ a one-dimensional caricature of a system of dimeric molecules. It has an absolutely continuous diffraction spectrum and an extra point in the dynamical spectrum (for the $\mathbb{Z}$-action of the discrete shift), which is due to the presence of a superstructure with periodic long-range order. One can recover this extra spectral information by considering the diffraction of a factor of the original system. Our example illustrates the distinction between dynamical and diffraction spectrum in a particularly simple manner.

Let us begin by briefly summarising the basic notions and concepts from diffraction theory and dynamical systems (we refer to [27, 28] and references therein for background on the dynamical systems used here). In our context, it is best to use a measure-theoretic setting for the systems under study, where we rely on the Riesz-Markov representation theorem to identify regular Borel measures on $\mathbb{R}^{d}$ with (continuous) linear functionals on the space $C_{\mathrm{c}}\left(\mathbb{R}^{d}\right)$ of continuous functions on $\mathbb{R}^{d}$ with compact support. In particular, we consider unbounded, complex measures $\omega$ on Euclidean space $\mathbb{R}^{d}$ that are translation bounded, which means that, for each compact set $K \subset \mathbb{R}^{d}$, we have $\sup _{t \in \mathbb{R}^{d}}|\omega|(t+K)<\infty$; see [14] for background. Such an $\omega$ describes the realisation of an infinite system, be it a crystal, a quasicrystal or a more general object.

Given such an $\omega$, let $\widetilde{\omega}$ be obtained from $\omega$ by reflection in the origin followed by complex conjugation, so that $\widetilde{\omega}(g)=\overline{\omega(\widetilde{g})}$ for any continuous function $g$ of compact support, where $\widetilde{g}$ is defined via $\widetilde{g}(x)=\overline{g(-x)}$. Given $\omega$, the corresponding autocorrelation measure $\gamma=\gamma_{\omega}$, or autocorrelation for short, is defined as the volume-averaged (or Eberlein [19]) convolution

$$
\gamma=\omega \circledast \widetilde{\omega}:=\lim _{R \rightarrow \infty} \frac{\left.\omega\right|_{R} * \widetilde{\left.\omega\right|_{R}}}{\operatorname{vol}\left(B_{R}(0)\right)},
$$

where $B_{R}(x)$ is the open ball of radius $R$ and centre $x$, while $\left.\omega\right|_{R}$ denotes the restriction of $\omega$ to the ball $B_{R}(0)$. The limit in (1) is taken in the vague topology, and will exist in all examples considered later, at least almost surely in the probabilistic sense; for some general results, see [21].

The measure $\gamma$ is positive definite by construction, and hence Fourier transformable. This gives $\widehat{\gamma}$, the diffraction measure or diffraction for short, which is a positive measure on $\mathbb{R}^{d}$ that describes the outcome of kinematic diffraction from $\omega$; see [15] for background and physical applications. The diffraction has a unique decomposition as

$$
\widehat{\gamma}=(\widehat{\gamma})_{\mathrm{pp}}+(\widehat{\gamma})_{\mathrm{sc}}+(\widehat{\gamma})_{\mathrm{ac}}
$$


into its pure point, singular continuous and absolutely continuous parts, where the decomposition of the continuous part is relative to Lebesgue measure. This is the Haar measure on $\mathbb{R}^{d}$ and also the right reference measure from the physical applications point of view.

A measure $\omega$ is called pure point diffractive when the corresponding diffraction measure satisfies $\widehat{\gamma}=(\widehat{\gamma})_{\mathrm{pp}}$, and similar definitions apply to the other spectral components. Important examples for pure point diffractive systems are perfect crystals and model sets [10,11,21], while the Thue-Morse sequence or the Rudin-Shapiro sequence, both with balanced weights, are paradigms for systems with purely singular continuous or purely absolutely continuous diffraction spectra; see $[5,6,17,22,29,31]$ and references therein for more. Here and below, a point set $\Lambda \subset \mathbb{R}^{d}$ is considered as a measure on $\mathbb{R}^{d}$ via its Dirac comb $\delta_{\Lambda}:=\sum_{x \in \Lambda} \delta_{x}$, and a sequence $\left(w_{n}\right)_{n \in \mathbb{Z}}$ as a measure on $\mathbb{Z}$ or on $\mathbb{R}$ (or both) via the weighted Dirac comb $\omega=\sum_{n \in \mathbb{Z}} w_{n} \delta_{n}$, where $\delta_{x}$ is the normalised point (or Dirac) measure at $x$.

In general, it is not adequate to restrict the attention to a single measure $\omega$. Equally relevant are translates of it, written as $\delta_{t} * \omega$, or any other measure that can be approximated arbitrarily well (in the vague topology) by such translates. Thus, one defines the hull $\mathbb{X}_{\omega}$ of $\omega$ as its vague orbit closure under the action of $\mathbb{R}^{d}$,

$$
\mathbb{X}_{\omega}=\overline{\left\{\delta_{t} * \omega \mid t \in \mathbb{R}^{d}\right\}}
$$

It is clear that the $\mathbb{R}^{d}$-action is continuous on $\mathbb{X}_{\omega}$, so that $\left(\mathbb{X}_{\omega}, \mathbb{R}^{d}\right)$ is a topological dynamical system. Since $\omega$ is assumed to be translation bounded, the hull $\mathbb{X}_{\omega}$ is compact in the vague topology [30]. More generally, we will consider a compact space $\mathbb{X}$ that contains the orbit closure $\mathbb{X}_{\omega}$ and emerges as the ensemble of possible realisations of an ergodic stochastic process.

We equip $\mathbb{X}$ with a translation invariant probability measure $\mu$ (which exists by standard arguments), and consider the (measure-theoretic) dynamical system $(\mathbb{X}, \mathcal{B}, \mu)$, where $\mathcal{B}$ is the standard Borel $\sigma$-algebra induced by the vague topology; see [16] for background. The measure $\mu$ also permits to consider $(\mathbb{X}, \mu)$ as a stochastic process $[3,17,20]$, as will be done below, too. The action of $\mathbb{R}^{d}$ now induces a unitary action on the Hilbert space $\mathrm{L}^{2}(\mathbb{X}, \mu)$. If the simultaneous eigenfunctions of the generators of the $\mathbb{R}^{d}$-action span $\mathrm{L}^{2}(\mathbb{X}, \mu)$, one speaks of pure point dynamical spectrum. In general, as before, one can have different spectral types, and one interesting question is the relation between the diffraction and the dynamical spectrum.

What follows, is an attempt to improve this situation by way of some guiding examples. Our focus will be on systems with some absolutely continuous spectrum, as the interest in them has recently been on the increase [34]. Afterwards, we summarise some general observations and formulate a more systematic connection between diffraction and dynamical spectrum.

\section{A Periodic Toy Model}

All our examples below are built on closed subsets of $\{ \pm 1\}^{\mathbb{Z}}$, which is compact in the obvious product topology. Let us begin with a quick glance at the set

$$
\mathbb{X}_{0}=\{\cdots-+-|+-+\cdots, \cdots+-+|-+-\cdots\}
$$

that consists of the two possible (truly) 2-periodic sequences within $\{ \pm 1\}^{\mathbb{Z}}$. Here and below, we use the shorthand \pm for \pm 1 and write a bi-infinite sequence as $w=\ldots w_{-2} w_{-1} \mid w_{0} w_{1} \ldots$, where $\mid$ denotes the origin. Giving each element of $\mathbb{X}_{0}$ probability $1 / 2$ defines $\mu_{0}$, the only 
possible invariant probability measure on $\mathbb{X}_{0}$, which is thus ergodic. The corresponding dynamical system $\left(\mathbb{X}_{0}, \mathcal{P}\left(\mathbb{X}_{0}\right), \mu_{0}\right)$, with $\mathcal{P}(A)$ denoting the power set of $A$, is clearly minimal, hence strictly ergodic; see [33] for general background.

Remark 1 At this stage, we only consider the $\mathbb{Z}$-action induced by the discrete shift operator. Its suspension into a dynamical system under the action of the full translation group $\mathbb{R}$ can later be added as a second step. It is trivial in the sense that one only sees the structure of the unit circle $\mathbb{S}$ in addition, in line with $\mathbb{R} / \mathbb{Z} \simeq \mathbb{S}$. In contrast, the diffraction measures below are always measures on $\mathbb{R}$.

Considering any $w \in \mathbb{X}_{0}$ and turning it into a Dirac comb (viewed as a tempered measure on $\mathbb{R}$ ) via

$$
\omega=\sum_{m \in \mathbb{Z}} h_{w(m)} \delta_{m}
$$

with arbitrary complex weights $h_{ \pm}$, one quickly checks by routine calculations that the corresponding autocorrelation in both cases reads

$$
\gamma=\frac{\left|h_{+}+h_{-}\right|^{2}}{4} \delta_{\mathbb{Z}}+\frac{\left|h_{+}-h_{-}\right|^{2}}{4}\left(\delta_{2 \mathbb{Z}}-\delta_{2 \mathbb{Z}+1}\right),
$$

with diffraction measure

$$
\widehat{\gamma}=\frac{\left|h_{+}+h_{-}\right|^{2}}{4} \delta_{\mathbb{Z}}+\frac{\left|h_{+}-h_{-}\right|^{2}}{4} \delta_{(2 \mathbb{Z}+1) / 2} .
$$

Note that $\gamma$ and $\widehat{\gamma}$ are to be understood as measures on $\mathbb{R}$. The last formula follows from an application of the Poisson summation formula for lattice Dirac combs [11], which reads

$$
\widehat{\delta_{\Gamma}}=\operatorname{dens}(\Gamma) \delta_{\Gamma^{*}}
$$

for a lattice $\Gamma$ and its dual lattice $\Gamma^{*}$. As expected for a periodic structure, $\widehat{\gamma}$ is a pure point measure. Since the original measure $\omega$ is supported on $\mathbb{Z}$, its diffraction is 1-periodic [2]. The 2-periodicity of $\omega$ in turn results in $\mathbb{Z} / 2$ as the support of $\widehat{\gamma}$. The latter can alternatively be written as

$$
\widehat{\gamma}=\frac{1}{4}\left(\left|h_{+}+h_{-}\right|^{2} \delta_{0}+\left|h_{+}-h_{-}\right|^{2} \delta_{1 / 2}\right) * \delta_{\mathbb{Z}},
$$

which illustrates both aspects. In particular, when $h_{ \pm}= \pm 1$, one obtains $\widehat{\gamma}=\delta_{\mathbb{Z}+\frac{1}{2}}$.

Let us compare this with the dynamical spectrum (under the $\mathbb{Z}$-action of the shift). Here, we clearly have $\mathrm{L}^{2}\left(\mathbb{X}_{0}, \mu_{0}\right) \simeq \mathbb{C}^{2}$, and there are two eigenfunctions, $f \equiv 1$ (for eigenvalue 1 ) and $g$ (for eigenvalue -1 ), the latter defined by $w \mapsto g(w)=w_{0}$. Together, they form an orthonormal basis of $\mathbb{C}^{2}$, relative to the inner product $\langle f \mid g\rangle=\int_{\mathbb{X}_{0}} \bar{f} g \mathrm{~d} \mu$. So, this is a simple example where one clearly sees how pure point diffraction spectrum and pure point dynamical spectrum fit together (and are, in fact, equivalent $[8,25,26]$ ). We will explain this in more detail later on.

\section{Close-packed Dimers on the Line with Random Orientation}

Consider close-packed dimers on the integers, at this stage viewed as empty boxes of length 2 that cover $\mathbb{Z}$ without overlaps or gaps. There are two possible configurations, which are 
distinguished by the central box (the latter either occupying the positions 0 and 1 , or -1 and 0 ). Let us now fill the boxes randomly with dimeric 'molecules', by distributing weights \pm 1 such that each dimer carries a 1 and a -1 , but in random order (or orientation). So, each box is then either $(+,-)$ or $(-,+)$. The result is a sequence in $\{ \pm 1\}^{\mathbb{Z}}$, where we now disregard the boxes again (they can always be reconstructed from a given sequence unless it is one of the two periodic sequences from $\mathbb{X}_{0}$ ).

The ensemble of all dimeric sequences as described above forms a closed and compact shift space $\mathbb{X}$, with a continuous action of the group $\mathbb{Z}$ via the usual shift operation. We call it the dimeric molecule shift, or DMS for short. More precisely, the shift $S: \mathbb{X} \longrightarrow \mathbb{X}$, defined by $(S w)_{n}=w_{n+1}$, is a continuous automorphism on $\mathbb{X}$ and generates an action of $\mathbb{Z}$. We will come back to this point of view shortly.

Consider a sequence $w \in \mathbb{X}$ and form the corresponding weighted Dirac comb

$$
\omega=w \delta_{\mathbb{Z}}:=\sum_{m \in \mathbb{Z}} w_{m} \delta_{m}
$$

which is a translation bounded (signed) measure on $\mathbb{Z}$ (and also on $\mathbb{R}$, via the canonical embedding of $\mathbb{Z}$ in $\mathbb{R}$ ). The corresponding autocorrelation measure (if it exists) is of the form $\gamma=\sum_{n \in \mathbb{Z}} \eta(n) \delta_{n}$, where the coefficients are given by the limits

$$
\eta(n)=\lim _{N \rightarrow \infty} \frac{1}{2 N+1} \sum_{m=-N}^{N} \overline{w_{m}} w_{m+n},
$$

provided the latter exist.

Lemma 1 For any $n \in \mathbb{Z}$, the autocorrelation coefficient $\eta(n)$ of the close-packed dimer model on $\mathbb{Z}$ with random orientation is given by

$$
\eta(n)= \begin{cases}1, & n=0 \\ -\frac{1}{2}, & n= \pm 1 \\ 0, & \text { otherwise. }\end{cases}
$$

Consequently, the autocorrelation is $\gamma=\delta_{0}-\frac{1}{2}\left(\delta_{1}+\delta_{-1}\right)$, which applies to almost all realisations of the DMS process.

Proof The process is clearly stationary and ergodic, so that we can determine $\eta$ from a typical realisation. One has $\eta(0)=1$ for every realisation, and $\eta(n)=\eta(-n)$ for $n \in \mathbb{Z}$ is clear whenever one of the coefficients exist. So, it remains to show the claim for $n \in \mathbb{N}$.

Since the sequences with all dimers in the same orientation form a null set, we may assume that at least one position $i \in \mathbb{Z}$ exists such that $w_{i}=w_{i+1}$. In a typical realisation, we have infinitely many such positions in the sequence, and they are either all even or all odd. We will use this structure implicitly in what follows.

When $n \geq 2$, the values $w_{m}$ and $w_{m+n}$ are independent. Moreover, the sum in (6) can be split into four sums, each of which is a sum over i.i.d. random variables of Bernoulli type. The strong law of large numbers (SLLN; see [18] for instance for a formulation with the slightly weaker assumption of mere pairwise independence, which will come in handy later on) then tells us that each contribution almost surely vanishes, so that $\eta(n)=0$ in this case.

In the remaining case $(n=1)$, every second term is -1 due to the structure of the dimers, which sums to $-\frac{1}{2}$. The remaining terms are the ones that cross the dimer boundaries, hence 
contribute 1 or -1 with equal probability and thus (almost surely) do not contribute to the overall sum (again by the SLLN). This gives $\eta(1)=-\frac{1}{2}$ and the proof is complete.

The corresponding diffraction follows by a straight-forward calculation.

Proposition 2 The diffraction of the DMS model is given by

$$
\widehat{\gamma}=(1-c) \lambda
$$

with $c(k)=\cos (2 \pi k)$, so that the measure $\widehat{\gamma}$ is absolutely continuous with respect to Lebesgue measure $\lambda$.

This particularly simple result is due to the balanced nature of the weights, so that

$$
\lim _{N \rightarrow \infty} \frac{1}{2 N+1} \sum_{n=-N}^{N} w_{n}=0
$$

holds for all realisations of our process. Let us now consider general weights, which we realise via the mapping $h:\{ \pm 1\} \longrightarrow \mathbb{C}$ that takes values $h_{ \pm}$. Given a realisation $w$, the new Dirac comb is then

$$
\omega_{h}=\sum_{m \in \mathbb{Z}} h\left(w_{m}\right) \delta_{m}=\frac{h_{+}+h_{-}}{2} \delta_{\mathbb{Z}}+\frac{h_{+}-h_{-}}{2} \omega,
$$

where $\omega$ is the Dirac comb of (5). The new autocorrelation turns out to be

$$
\gamma_{h}=\omega_{h} \circledast \widetilde{\omega_{h}}=\frac{\left|h_{+}+h_{-}\right|^{2}}{4} \delta_{\mathbb{Z}}+\frac{\left|h_{+}-h_{-}\right|^{2}}{4} \gamma
$$

with the $\gamma$ from the balanced weight case of Lemma 1. Note that (9) holds almost surely (as $\gamma$ does), and rests upon $\delta_{\mathbb{Z}} \circledast \widetilde{\delta_{\mathbb{Z}}}=\delta_{\mathbb{Z}}$ together with $\delta_{\mathbb{Z}} \circledast \widetilde{\omega}=0$ and $\widetilde{\delta_{\mathbb{Z}}} \circledast \omega=0$. The latter two identities are a consequence of (7).

The corresponding diffraction measure $\widehat{\gamma_{h}}$ can be calculated via Fourier transform and an application of Proposition 2. It (almost surely) reads

$$
\widehat{\gamma_{h}}=\frac{\left|h_{+}+h_{-}\right|^{2}}{4} \delta_{\mathbb{Z}}+\frac{\left|h_{+}-h_{-}\right|^{2}}{4}(1-c) \lambda,
$$

with $c(k)=\cos (2 \pi k)$ as above. This is a measure of mixed type, with a pure point part and an absolutely continuous one. However, the point part is trivial in the sense that it only reflects the lattice support of the Dirac comb $\omega_{h}$ and thus does not carry any other relevant information on the system. This corresponds to the trivial (constant) eigenfunction of the dynamical spectrum, which we determine next.

\section{The DMS and Its Dynamical Spectrum}

Let us look at the above model from the viewpoint of dynamical systems. As before, we begin with the $\mathbb{Z}$-action of the shift operator. Given a sequence $w \in\{ \pm 1\}^{\mathbb{Z}}$, let us first define

$$
M(w)=\left\{m \in \mathbb{Z} \mid w_{m}=w_{m+1}\right\} .
$$


Note that $M(w)=\varnothing$ precisely for $w \in \mathbb{X}_{0}$, with $\mathbb{X}_{0}$ from (3). The set of all dimeric molecule sequences from Sect. 3 forms the ensemble

$$
\mathbb{X}=\left\{w \in\{ \pm 1\}^{\mathbb{Z}} \mid M(w) \subset 2 \mathbb{Z} \text { or } M(w) \subset 2 \mathbb{Z}+1\right\},
$$

which is a closed subshift (and hence a compact set). We call a sequence even (odd) when $M(w)$ is non-empty and a subset of $2 \mathbb{Z}($ of $2 \mathbb{Z}+1)$. Then, $\mathbb{X}$ splits as

$$
\mathbb{X}=\mathbb{X}_{+} \dot{\cup} \mathbb{X}_{-} \dot{U} \mathbb{X}_{0}
$$

where $\mathbb{X}_{+}$and $\mathbb{X}_{-}$denote the closed subsets of even and odd sequences, respectively, while $\dot{U}$ denotes the disjoint union of sets.

The shift $S$ (as defined earlier) is viewed as the (continuous) generator of the action of $\mathbb{Z}$ on $\{ \pm 1\}^{\mathbb{Z}}$. As $\mathbb{X}$ is clearly shift-invariant, we obtain $(\mathbb{X}, S)$ as a topological dynamical system (with $\mathbb{Z}$-action). It is clear from standard arguments that there are invariant probability measures on $\mathbb{X}$. Indeed, the underlying process highlights a natural choice for a measure $\mu$, as is also clear from the proof of Lemma 1 . It satisfies $\mu\left(\mathbb{X}_{0}\right)=0$ together with $\mu\left(\mathbb{X}_{+}\right)=\mu\left(\mathbb{X}_{-}\right)=\frac{1}{2}$. Within $\mathbb{X}_{+}$, each dimer then has equal probability to be either $(+,-)$ or $(-,+)$, so that the corresponding cylinder sets and their measures are well-defined. We can now view $\left(\mathbb{X}, \mathcal{B}_{\mathbb{X}}, \mu\right)$ as a measure-theoretic dynamical system (under the action of $\mathbb{Z}$ via the shift $S$ ), where $\mathcal{B}_{\mathbb{X}}$ is the standard Borel $\sigma$-algebra on $\mathbb{X}$.

Let us next consider the Hilbert space $\mathcal{H}=\mathrm{L}^{2}(\mathbb{X}, \mu)$, with the induced action of $S$ via $U: \mathcal{H} \longrightarrow \mathcal{H}$, as defined by $f \mapsto U f$ with $U f(w):=f(S w)$. The inner product is written as

$$
\langle f \mid g\rangle=\int_{\mathbb{X}} \overline{f(w)} g(w) \mathrm{d} \mu(w),
$$

where $\langle U f \mid U g\rangle=\langle f \mid g\rangle$ holds due to the shift invariance of $\mu$. In fact, $U$ is unitary.

The function $\varphi \equiv 1$ is an eigenfunction of $U$ with eigenvalue 1 as usual, but we also have an eigenfunction for the eigenvalue -1 , namely the one defined by

$$
\psi(w)= \begin{cases}0, & \text { if } w \in \mathbb{X}_{0}, \\ \pm 1, & \text { if } w \in \mathbb{X}_{ \pm},\end{cases}
$$

which is well-defined because $\mathbb{X}_{0}, \mathbb{X}_{+}$and $\mathbb{X}_{-}$are measurable sets. Clearly, $\langle\varphi \mid \psi\rangle=0$, since $\mu\left(\mathbb{X}_{+}\right)=\mu\left(\mathbb{X}_{-}\right)=\frac{1}{2}$, while $\psi^{2}=\varphi$ holds $\mu$-almost everywhere. Note also that $\psi$ can be written as a limit via

$$
\psi(w)=\lim _{N \rightarrow \infty} \frac{2}{2 N+1} \sum_{n=-N}^{N}(-1)^{n} w_{n} w_{n+1},
$$

which exists for $\mu$-almost all $w \in \mathbb{X}$ by an SLLN argument analogous to that used in the proof of Lemma 1.

So far, we know that the $\mathbb{C}$-span of $\varphi$ and $\psi$ is contained in $\mathcal{H}_{\mathrm{pp}}$. To see that we actually have equality here, consider the double shift $S^{2}$ on $\mathbb{X}$. First, for any $w \in \mathbb{X}$, the entries $w_{n}$ and $w_{n+2}$ are independent, so that, for any fixed $i \in \mathbb{Z}$, the sequence $\left(S^{2 n} w\right)_{i}$ with $n \in \mathbb{Z}$ is a coin tossing sequence. Consequently, $S^{2}$ must comprise a spectrum of countable Lebesgue type. On the other hand, for any given $w$, the sequences with indeces $i$ and $i+1$ are dependent, which is reflected by the fact that $\mathbb{X}$ is not minimal for the action of $S^{2}$. Here, $\mathbb{X}_{0}, \mathbb{X}_{+}$ and $\mathbb{X}_{-}$are the non-trivial invariant subspaces. They lead to two eigenfunctions of $S^{2}$ with 
eigenvalue 1 , namely the characteristic functions $\mathbf{1}_{+}$and $\mathbf{1}_{-}$(on $\mathbb{X}_{+}$and $\mathbb{X}_{-}$), with $\varphi=\mathbf{1}_{+}+$ $\mathbf{1}_{-}$and $\psi=\mathbf{1}_{+}-\mathbf{1}_{-}$(both holding $\mu$-almost everywhere). Our system is one-dependent in the sense of [1], and can be viewed as the average of two Bernoulli shifts. Consequently, 1 is the only eigenvalue of $S^{2}$, and no singular continuous contribution exists. The spectral theorem now tells us that $S$ has precisely the two eigenfunctions constructed above and only absolutely continuous spectrum otherwise. In particular, we have no freedom for singular continuous components.

Let us now expand on the continuous part of the spectrum. To this end, we consider the function defined by $w \mapsto \sigma_{n}(w)=w_{n}+w_{n+1}$, which is continuous on $\mathbb{X}$ and hence measurable. A short calculation reveals that

$$
\left\langle\varphi \mid \sigma_{n}\right\rangle=\left\langle\psi \mid \sigma_{n}\right\rangle=0
$$

so that $\sigma_{n} \in \mathcal{H}_{\mathrm{pp}}^{\perp}$, while $U \sigma_{n}=\sigma_{n+1}$. The smallest $U$-invariant subspace of $\mathcal{H}$ that contains $\sigma_{n}$ is thus the cyclic space

$$
\mathcal{C}\left(\sigma_{n}\right)=\bigoplus_{m \in \mathbb{Z}} \mathbb{C} \sigma_{m}
$$

For any $m \in \mathbb{Z}$, the spectral measure of $\sigma_{m}$ is given by

$$
\begin{aligned}
\left\langle\sigma_{m} \mid U^{n} \sigma_{m}\right\rangle & =\int_{\mathbb{X}}\left(w_{m}+w_{m+1}\right)\left(w_{m+n}+w_{m+n+1}\right) \mathrm{d} \mu(w) \\
& =\delta_{n, 0}-\frac{1}{2}\left(\delta_{n, 2}+\delta_{n,-2}\right),
\end{aligned}
$$

which is a positive definite function on $\mathbb{Z}$. In its calculation, we have used

$$
\int_{\mathbb{X}} w_{m} w_{m+k} \mathrm{~d} \mu(w)= \begin{cases}1, & \text { if } k=0 \\ -\frac{1}{2}, & \text { if }|k|=1 \\ 0, & \text { if }|k| \geq 2\end{cases}
$$

which follows by an argument used before for the determination of the autocorrelation coefficients $\eta(m)$ in Lemma 1.

By the Herglotz-Bochner theorem, the positive definite function $n \mapsto\left\langle\sigma_{m} \mid U^{n} \sigma_{m}\right\rangle$ is the Fourier transform of a positive measure, which means that

$$
\left\langle\sigma_{m} \mid U^{n} \sigma_{m}\right\rangle=\int_{0}^{1} e^{-2 \pi i n x} \mathrm{~d} \nu_{m}(x)
$$

for some positive measure $v_{m}$ on the unit circle $\mathbb{S}$, represented here by the unit interval (with periodic boundary conditions). By routine calculation, one finds

$$
v_{m}=\lambda-\frac{1}{2}\left(e^{4 \pi i k}+e^{-4 \pi i k}\right) \lambda=(1-\cos (4 \pi k)) \lambda,
$$

where the bracketed factor is the Radon-Nikodym density of $v_{m}$ relative to Lebesgue measure $\lambda$, written as a function of the variable $k$ (which is equivalent to our previous formulation of a density, as used in Proposition 2 and (10)). In particular, $v_{m}$ is absolutely continuous (relative to $\lambda$ ), and does not depend on $m$. Note that $v_{m}$ is obtained from the diffraction measure by doubling the argument in its Radon-Nikodym density. 
This is one of countably many mutually orthogonal cyclic spaces with absolutely continuous spectral measures, as once again follows from the underlying Bernoulli structure. Together with the two eigenvalues and the absence of a singular continuous part, we have established the following result.

Proposition 3 The dynamical spectrum of the DMS under $\mathbb{Z}$-action is a mixture of a pure point part and an absolutely continuous one. The eigenvalues are \pm 1 , while the remainder is of countable Lebesgue type.

So far, we have formulated the spectrum 'naively', without any reference to harmonic analysis and duality. It is more systematic to include the dual group to $\mathbb{Z}$ into the picture, which is the unit circle $\mathbb{S}$, conveniently represented by the half-open interval $[0,1)$ together with addition modulo 1 . Then, one sees (via the elements of the dual group as characters on $\mathbb{Z}$ ) that our eigenvalues 1 and -1 correspond to the elements 0 and $\frac{1}{2}$ of the unit circle, with $\frac{1}{2}$ being the non-trivial contribution.

This point of view is particularly useful when we suspend the $\mathbb{Z}$-action into the continuous translation action of the group $\mathbb{R}$, as given by $\omega \mapsto \delta_{t} * \omega$ with $t \in \mathbb{R}$. Now, the dual group is $\mathbb{R}$ (since $\mathbb{R}$ is self-dual), and our dynamical spectrum becomes $\mathbb{Z} / 2$. This is the natural formulation for the comparison with the (support of the) diffraction spectrum. The pure point part of the dynamical spectrum is non-trivial because it is $\mathbb{Z} / 2$ rather than $\mathbb{Z}$.

At this point, we note that the relation between the dynamical and the diffraction spectrum is reminiscent of the situation for the Thue-Morse sequence. In both cases, there exists some non-trivial point spectrum that is not reflected in the diffraction measure of the sequence. However, the missing spectral part of the Thue-Morse sequence is retrieved via the period doubling sequence, which (as a dynamical system) can be viewed as a factor the Thue-Morse system. We will now demonstrate that the analogous situation is also met for our new example.

\section{A Factor System and Its Diffraction}

Let us define a mapping $\phi: \mathbb{X} \longrightarrow\{ \pm 1\}^{\mathbb{Z}}$ via $w \mapsto \phi(w)$ with

$$
\phi(w)_{n}=-w_{n} w_{n+1},
$$

which is continuous. This particular mapping is inspired by the analogous situation for the Thue-Morse sequence, and indeed has similar consequences here. The image set, $\mathbb{Y}=\phi(\mathbb{X})$, is again compact, and $(\mathbb{Y}, S)$ is another topological dynamical system. It is clear that $\phi(-w)=\phi(w)$, where $(-w)_{n}=-w_{n}$, and a moments reflection shows that this is the only ambiguity, so that $\phi: \mathbb{X} \longrightarrow \mathbb{Y}$ is a globally two-to-one surjection. In particular, $\mathbb{Y}_{0}=\phi\left(\mathbb{X}_{0}\right)=\{\ldots 11 \mid 11 \ldots\}$, and the entire image shift space is

$$
\mathbb{Y}=\left\{v \in\{ \pm 1\}^{\mathbb{Z}} \mid v_{n}=1 \text { for all } n \in 2 \mathbb{Z} \text { or for all } n \in 2 \mathbb{Z}+1\right\}
$$

Moreover, $\phi$ makes the diagram

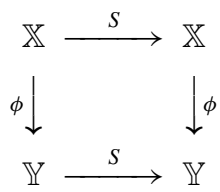


commutative. Consequently, $(\mathbb{Y}, S)$ is a (topological) factor [16] of the dynamical system $(\mathbb{X}, S)$. In our setting, the measure $\mu$ on $\mathbb{X}$ induces a measure $v$ on $\mathbb{Y}$ via $v(A)=$ $(\phi . \mu)(A):=\mu\left(\phi^{-1}(A)\right)$ for Borel sets $A$. We may thus also consider the dynamical system $\left(\mathbb{Y}, \mathcal{B}_{\mathbb{Y}}, v\right)$, which is then a measure-theoretic factor of $\left(\mathbb{X}, \mathcal{B}_{\mathbb{X}}, \mu\right)$; see [9, Sect. 3] for a summary of factors and their spectral properties.

Let us first look at diffraction, for a typical element $v \in \mathbb{Y}$.

Lemma 4 The autocorrelation coefficients of $v \in \mathbb{Y}$ are $v$-almost surely given by $\eta(0)=1$, $\eta(2 n)=\frac{1}{2}$ for all $n \in \mathbb{Z} \backslash\{0\}$, and $\eta(2 m+1)=0$ for all $m \in \mathbb{Z}$.

Proof The mapping $\phi$ has the effect that a typical $v \in \mathbb{Y}$ has weights 1 on every second position, and weights \pm 1 with equal probability on all remaining positions. The latter form an i.i.d. family of random variables, so that an application of the SLLN, in the same spirit as used before, gives the formula for $\eta$.

Proposition 5 Autocorrelation and diffraction of $\mathbb{Y}$ are given by

$$
\gamma=\frac{1}{2} \delta_{0}+\frac{1}{2} \delta_{2 \mathbb{Z}} \quad \text { and } \quad \widehat{\gamma}=\frac{1}{2} \lambda+\frac{1}{4} \delta_{\mathbb{Z} / 2}
$$

which apply to almost all realisations of the underlying process.

In particular, the diffraction is of mixed type, with a non-trivial pure point component.

Proof The claim on $\gamma$ is clear from Lemma 4, while its Fourier transform follows from $\widehat{\delta_{0}}=\lambda$ together with an application of the Poisson summation formula to $\delta_{2 \mathbb{Z}}$.

As before, this is the result for weights \pm 1 . Since they are no longer balanced, the calculation of the diffraction formula for general weights needs one extra step. Observing that

$$
\lim _{N \rightarrow \infty} \frac{1}{2 N+1} \sum_{n=-N}^{N} v_{n}=\frac{1}{2}
$$

holds for $v$-almost all $v \in \mathbb{Y}$, one easily derives the Eberlein convolutions

$$
\delta_{\mathbb{Z}} \circledast \widetilde{\omega}=\frac{1}{2} \delta_{\mathbb{Z}} \quad \text { and } \quad \omega \circledast \widetilde{\delta_{\mathbb{Z}}}=\frac{1}{2} \delta_{\mathbb{Z}},
$$

which apply $v$-almost surely. Since the general Dirac comb $\omega_{h}$ again satisfies (8), a simple calculation results in

$$
\gamma_{h}=\frac{\left|h_{+}+h_{-}\right|^{2}+\left|h_{+}\right|^{2}-\left|h_{-}\right|^{2}}{4} \delta_{\mathbb{Z}}+\frac{\left|h_{+}-h_{-}\right|^{2}}{4} \gamma
$$

and thus in the general diffraction formula

$$
\widehat{\gamma_{h}}=\frac{\left|h_{+}+h_{-}\right|^{2}+\left|h_{+}\right|^{2}-\left|h_{-}\right|^{2}}{4} \delta_{\mathbb{Z}}+\frac{\left|h_{+}-h_{-}\right|^{2}}{16} \delta_{\mathbb{Z} / 2}+\frac{\left|h_{+}-h_{-}\right|^{2}}{8} \lambda,
$$

by an application of Proposition 5. The intensity of any point measure $\delta_{k}$ with $k \in \mathbb{Z}$ is thus given by $\left|\frac{3}{4} h_{+}+\frac{1}{4} h_{-}\right|^{2}$, which is the absolute square of the average weight (or scattering 
strength) in this case, as it must. Equation (17) displays a non-trivial pure point component (namely the one with support $\mathbb{Z} / 2$ ) that 'recovers' the missing part from our original dynamical system $\left(\mathbb{X}, \mathcal{B}_{\mathbb{X}}, \mu\right)$.

The dynamical spectrum of $\left(\mathbb{Y}, \mathcal{B}_{\mathbb{Y}}, v\right)$ is the same as that of $\left(\mathbb{X}, \mathcal{B}_{\mathbb{X}}, \mu\right)$. Given an element $v \in \mathbb{Y}$, our previous eigenfunction $g$ takes the same value on the two pre-images in $\phi^{-1}(v)$, so that we once again have an eigenfunction for the eigenvalue -1 , in addition to the constant function (for the eigenvalue 1). The Bernoulli substructure is responsible for a continuous spectrum of countable Lebesgue type. Note that a factor system need not have the same dynamical spectrum as the original system, as the example of the Thue-Morse sequence versus the period doubling sequence demonstrates.

Remark 2 There is another very simple (albeit somewhat degenerate) possibility to define a factor. Recall that $\mathbb{X}_{0}=\left\{u_{+}, u_{-}\right\}$with $u_{+}=\cdots+-\mid+-\cdots$ and $u_{-}=S u_{+}$. Now, define the mapping $\psi: \mathbb{X} \longrightarrow \mathbb{X}_{0}$ by

$$
w \longmapsto \psi(w):= \begin{cases}w, & \text { if } w \in \mathbb{X}_{0}, \\ u_{ \pm}, & \text {if } w \in \mathbb{X}_{ \pm},\end{cases}
$$

which is a continuous surjection and shows that our toy system of Sect. 2 is a factor of the DMS. Here, the factor has pure point spectrum. The diffraction spectrum is $\mathbb{Z} / 2$, which exhausts the dynamical spectrum of the DMS.

Our observations up to this point can be summarised as follows.

Theorem 6 The diffraction measure of the DMS system with balanced weights is purely absolutely continuous, as stated in Proposition 2. The case of general weights can only add the trivial pure point part, as given in (10).

The dynamical spectrum of the DMS system under the action of $\mathbb{R}$ consists of the pure point part $\mathbb{Z} / 2$ together with a countable Lebesgue spectrum.

The non-trivial part $(\mathbb{Z} / 2) \backslash \mathbb{Z}$ of the dynamical point spectrum is not reflected by the diffraction spectrum of the DMS system, but can be recovered via the diffraction spectrum of a suitably chosen factor of it, either via the toy model of Sect. 2 and Remark 2 or via the system $\mathbb{Y}$ from Proposition 5.

Proof Most claims are clear from the previous propositions. The dynamical spectrum for the action of $\mathbb{R}$, which is written via the generating elements (so that $\frac{1}{2}$ corresponds to an eigenvalue -1 as explained earlier), follows from the spectrum for the $\mathbb{Z}$-action of the shift in Proposition 3 via a standard suspension. Since we deal with a realisation of the system as a set of Dirac combs with support $\mathbb{Z}$, this only extends the set $\left\{\frac{1}{2}, 1\right\}$ to the group generated by it, which is $\mathbb{Z} / 2$.

Up to this point, we can observe that the diffraction of $\mathbb{X}$ does not reflect the full dynamical spectrum of $\mathbb{X}$, while the diffraction of $\mathbb{X}$ together with that of its factor $\mathbb{Y}$ does.

The analogous phenomenon appears in the case of the Thue-Morse system [5, 17], where the dyadic rationals in the dynamical spectrum [27] are only recovered via the diffraction spectrum of the period doubling system, which is again a factor with pure point diffraction (in fact, it can be described as a 2-adic model set [7, 11, 12]). Let us thus look at this situation from a more general point of view. 


\section{General Observations and Outlook}

Here and below, $\mathbb{X}$ is a compact dynamical system of (possibly weighted) Dirac combs on $\mathbb{Z}^{d}$ or of translation bounded measures on $\mathbb{R}^{d}$, with ergodic invariant measure $\mu$ under the action of the translation group $\mathbb{Z}^{d}$ or $\mathbb{R}^{d}$. Let $\mathbb{Y}$ be a factor of $\mathbb{X}$, with factor map $\phi$ and induced measure $v$. In particular, we assume that diagram (16) is again commutative, with $S$ replaced by any generator of our translation group.

If $g$ is an eigenfunction in $\mathrm{L}^{2}(\mathbb{Y}, v)$, it is clear that $g \circ \phi \in \mathrm{L}^{2}(\mathbb{X}, \mu)$, and the commutativity of the diagram (16) implies that the latter is again an eigenfunction, with the same eigenvalue (or set of eigenvalues, if $d>1$ ).

Fact 7 The dynamical eigenvalues of the factor system $\mathbb{Y}$ form a subset of those of the original system $\mathbb{X}$.

This is one ingredient for the following result; see $[9,16]$ for more.

\section{Proposition 8 If $\mathbb{X}$ has pure point dynamical spectrum, then so does $\mathbb{Y}$.}

More generally, it seems difficult for a factor to decrease the long-range order in the diffraction, except for the removal of the pure point part that corresponds to the trivial eigenfunction (via the balanced weight representation). It is also rather clear that, by means of suitable correlation functions, one can detect each eigenfunction in the diffraction measure of a suitable factor. This is known explicitly for the Thue-Morse sequence, but also for the Rudin-Shapiro sequence, both having the dyadic rationals as the pure point part of the dynamical spectrum [27]. Once a factor is pure point, further factors can only reduce to subgroups, and hence do not contain new information on the system. This mechanism also underlies the equivalence of diffraction and dynamical spectrum in the pure point case.

However, it is less obvious that a factor could display a singular continuous diffraction spectrum if the original system does not. That this is indeed possible is once again visible from the TM sequences. Recall that the TM hull $\mathbb{X}_{\mathrm{TM}}$ can be defined via the primitive substitution $1 \mapsto 1 \overline{1}, \overline{1} \mapsto \overline{1} 1$ on the binary alphabet $\{1, \overline{1}\}$. For any $w \in \mathbb{X}_{\mathrm{TM}}$, replace 1 and $\overline{1}$ by the weights $\frac{1}{5}$ and $\frac{7}{5}$, followed by a random and independent choice of a sign $(+$ or -) for each weight. This way, one defines an (infinite) cover of $\mathbb{X}_{\mathrm{TM}}$. Each element of it has average squared scattering strength 1 , while each typical element has vanishing 2-point correlations. Consequently, the diffraction measure of the covering hull is $\widehat{\gamma}=\lambda$, which is purely absolutely continuous. The TM system, which is a factor, has purely singular continuous diffraction (for the balanced weight case), while the period doubling system, which is again a factor, is pure point. So, this little example illustrates a step-wise unravelling of the order phenomena.

Note that what we say here is more general than (and somewhat different from) the direct discussion of dynamical versus diffraction spectrum in a single system. Indeed, if the diffraction spectrum is pure point, then so is the dynamical spectrum. But it is certainly possible to have a factor with pure point diffraction spectrum when the original system has a dynamical spectrum with also continuous components-this is what the known examples demonstrate. Somehow, the dynamical spectrum contains the information of the diffraction spectra of all its factors. Conversely, in all known examples so far, the diffraction spectra of a system and its factors taken together seem to comprise the complete information on the dynamical spectrum of the original system (even though the diffraction spectrum of each individual factor might not be very informative at all). 
The general claim is rather clear now: The dynamical spectrum is not to be compared with the diffraction spectrum of the system alone, but with the diffraction spectra of the system and all its factors. A more general and precise formulation and exposition is postponed to a forthcoming publication [4].

Let us close by some remarks on the relation of our findings to some more general issues investigated in statistical physics. Our DMS model forms a caricature of a system where 'molecules' are ordered, but due to a disordered interior of each molecule, 'atoms' do not display long-range order. Although we have a 1-dimensional ground state order for the dimeric molecules, which is typical for $T=0$, and independent disorder on the 'atomic' level, which is typical for infinite temperatures, we expect conceptually similar phenomena to be rather widespread. In more realistic models, one should have a similar result for appropriate Gibbs measures, which then should be higher-dimensional. For some preliminary results on diffraction, mixing properties and spectra of equilibrium systems (as described by Gibbs measures), we refer to [13, 23, 24, 32].

Acknowledgements It is a pleasure to thank Roberto Fernández, Uwe Grimm, Daniel Lenz and Frank Redig for helpful discussions. We thank two reviewers for a number of very useful suggestions. This work was supported by the German Research Council (DFG), within the CRC 701.

Open Access This article is distributed under the terms of the Creative Commons Attribution Noncommercial License which permits any noncommercial use, distribution, and reproduction in any medium, provided the original author(s) and source are credited.

\section{References}

1. Aaronson, J., Gilat, D., Keane, M., de Valk, V.: An algebraic construction of a class of one-dependent processes. Ann. Probab. 17, 128-143 (1989)

2. Baake, M.: Diffraction of weighted lattice subsets. Can. Math. Bull. 45, 483-498 (2002). arXiv:math.MG/0106111

3. Baake, M., Birkner, M., Moody, R.V.: Diffraction of stochastic point sets: Explicitly computable examples. Commun. Math. Phys. 293, 611-660 (2009). arXiv:0803.1266

4. Baake, M., van Enter, A.C.D., Lenz, D.: On the relation between dynamical and diffraction spectra, in preparation

5. Baake, M., Grimm, U.: The singular continuous diffraction measure of the Thue-Morse chain. J. Phys. A, Math. Theor. 41, 422001 (2008). arXiv:0809.0580

6. Baake, M., Grimm, U.: Kinematic diffraction is insufficient to distinguish order from disorder. Phys. Rev. B 79, 020203(R) (2009) and Phys. Rev. B 80, 029903(E) (2009). arXiv:0810.5750

7. Baake, M., Grimm, U.: Diffraction of limit periodic point sets. Philos. Mag. (2010). doi:10.1080/ 14786435.2010.508447. arXiv:1007.0707

8. Baake, M., Lenz, D.: Dynamical systems on translation bounded measures: pure point dynamical and diffraction spectra. Ergod. Theory Dyn. Syst. 24, 1867-1893 (2004). math.DS/0302231

9. Baake, M., Lenz, D.: Deformation of Delone dynamical systems and topological conjugacy. J. Fourier Anal. Appl. 11, 125-150 (2005). math.DS/0404155

10. Baake, M., Lenz, D., Moody, R.V.: Characterization of model sets by dynamical systems. Ergod. Theory Dyn. Syst. 27, 341-382 (2007). arXiv:math/0511648

11. Baake, M., Moody, R.V.: Weighted Dirac combs with pure point diffraction. J. Reine Angew. Math. (Crelle) 573, 61-94 (2004). arXiv:math.MG/0203030

12. Baake, M., Moody, R.V., Schlottmann, M.: Limit-(quasi-)periodic point sets as quasicrystals with $p$-adic internal spaces. J. Phys. A, Math. Gen. 31, 5755-5765 (1998). math-ph/9901008

13. Baake, M., Sing, B.: Diffraction spectrum of lattices gas models above $T_{c}$. Lett. Math. Phys. 68, 165-173 (2004). math-ph/0405064

14. Berg, C., Forst, G.: Potential Theory on Locally Compact Abelian Groups. Springer, Berlin (1975)

15. Cowley, J.M.: Diffraction Physics, 3rd edn. North-Holland, Amsterdam (1995)

16. Denker, M., Grillenberger, C., Sigmund, K.: Ergodic Theory of Compact Spaces. LNM, vol. 527. Springer, Berlin (1976) 
17. van Enter, A.C.D., Miękisz, J.: How should one define a (weak) crystal? J. Stat. Phys. 66, 1147-1153 (1992)

18. Etemadi, N.: An elementary proof of the strong law of large numbers. Z. Wahrscheinlichkeitstheor. Verw. Geb. 55, 119-122 (1981)

19. Gil de Lamadrid, J., Argabright, L.N.: Almost Periodic Measures. Memoirs AMS, vol. 85(428). AMS, Providence (1990)

20. Goueré, J.-B.: Diffraction and Palm measure of point processes. C. R. Acad. Sci. (Paris) 342, 141-146 (2003). arXiv:math.PR/0208064

21. Hof, A.: On diffraction by aperiodic structures. Commun. Math. Phys. 169, 25-43 (1995)

22. Höffe, M., Baake, M.: Surprises in diffuse scattering. Z. Kristallogr. 215, 441-444 (2000). arXiv: math-ph/0004022

23. Külske, C.: Universal bounds on the selfaveraging of random diffraction measures. Probab. Theory Relat. Fields 126, 29-50 (2003). arXiv:math-ph/0109005

24. Külske, C.: Concentration inequalities for functions of Gibbs fields with application to diffraction and random Gibbs measures. Commun. Math. Phys. 239, 29-51 (2003)

25. Lee, J.-Y., Moody, R.V., Solomyak, B.: Pure point dynamical and diffraction spectra. Ann. Henri Poincaré 3, 1003-1018 (2002). arXiv:0910.4809

26. Lenz, D., Strungaru, N.: Pure point spectrum for measure dynamical systems on locally compact Abelian groups. J. Math. Pures Appl. 92, 323-341 (2009). arXiv:0704.2498

27. Queffélec, M.: Substitution Dynamical Systems-Spectral Analysis, 2nd edn. Springer, Berlin (2010)

28. Robinson, E.A. Jr.: Symbolic dynamics and tilings of $\mathbb{R}^{d}$. Proc. Symp. Appl. Math. 60, 81-119 (2004)

29. Rudin, W.: Some theorems on Fourier coefficients. Proc. Am. Math. Soc. 10, 855-859 (1959)

30. Schlottmann, M.: Generalised model sets and dynamical systems. In: Baake, M., Moody, R.V. (eds.) Directions in Mathematical Quasicrystals. CRM Monograph Series, vol. 13, pp. 143-159. AMS, Providence (2000)

31. Shapiro, H.: Extremal problems for polynomials and power series. Masters Thesis, MIT, Boston (1951)

32. Slawny, J.: Ergodic properties of equilibrium states. Commun. Math. Phys. 80, 477-483 (1981)

33. Walters, P.: An Introduction to Ergodic Theory. Springer, New York (2000). Reprint

34. Withers, R.L.: Disorder, structured diffuse scattering and the transmission electron microscope. Z. Kristallogr. 220, 1027-1034 (2005) 